\title{
Kardar-Parisi-Zhang Universality
}

Ivan Corwin (Columbia University, New York, USA)

\section{$1 \quad$ Universality in random systems}

Universality in complex random systems is a striking concept that has played a central role in the direction of research within probability, mathematical physics and statistical mechanics. In this article, we will describe how a variety of physical systems and mathematical models, including randomly growing interfaces, certain stochastic PDEs, traffic models, paths in random environments and random matrices, all demonstrate the same universal statistical behaviours in their long-time/large-scale limit. These systems are said to lie in the Kardar-Parisi-Zhang (KPZ) universality class. Proof of universality within these classes of systems (except for random matrices) has remained mostly elusive. Extensive computer simulations, non-rigorous physical arguments and heuristics, some laboratory experiments and limited mathematically rigorous results provide important evidence for this belief.

The last 15 years have seen a number of breakthroughs in the discovery and analysis of a handful of special integrable probability systems, which, due to enhanced algebraic structure, admit many exact computations and, ultimately, asymptotic analysis revealing the purportedly universal properties of the KPZ class. The structures present in these systems generally originate in representation theory (e.g., symmetric functions), quantum integrable systems (e.g. Bethe ansatz) and algebraic combinatorics (e.g., RSK correspondence) and the techniques in their asymptotic analysis generally involve Laplace's method, Fredholm determinants or Riemann-Hilbert problem asymptotics.

This article will focus on the phenomena associated with the KPZ universality class [3] and highlight how certain integrable examples expand the scope of and refine the notion of universality. We start by providing a brief introduction to the Gaussian universality class and the integrable probabilistic example of random coin flipping, as well as the random deposition model. A small perturbation to the random deposition model leads us to the ballistic deposition model and the KPZ universality class. The ballistic deposition model fails to be integrable; thus, to gain an understanding of its long-time behaviour and that of the entire KPZ class, we turn to the corner growth model. The rest of the article focuses on various sides of this rich model: its role as a random growth process, its relation to the KPZ stochastic PDE, its interpretation in terms of interacting particle systems and its relation to optimisation problems involving paths in random environments. Along the way, we include some other generalisations of this process whose integrability springs from the same sources. We close the article by reflecting upon some open problems.

A survey of the KPZ universality class and all of the associated phenomena and methods developed or utilised in its study is far too vast to be provided here. This article presents only one of many stories and perspectives regarding this rich area of study. To even provide a representative cross-section of references is beyond this scope. Additionally, even though we will discuss integrable examples, we will not describe the algebraic structures and methods of asymptotic analysis behind them (despite their obvious importance and interest). Some recent references that review some of these structures include $[2,4,8]$ and references therein. On the more physics-oriented side, the collection of reviews and books $[1,3,5,6,7,8,9,10]$ provides some idea of the scope of the study of the KPZ universality class and the diverse areas upon which it touches.

We start now by providing an overview of the general notion of universality in the context of the simplest and historically first example - fair coin flipping and the Gaussian universality class.

\section{Gaussian universality class}

Flip a fair coin $N$ times. Each string of outcomes (e.g., head, tail, tail, tail, head) has an equal probability $2^{-N}$. Call $H$ the (random) number of heads and let $\mathbb{P}$ denote the probability distribution for this sequence of coin flips. Counting shows that

$$
\mathbb{P}(H=n)=2^{-N}\left(\begin{array}{l}
N \\
n
\end{array}\right) .
$$

Since each flip is independent, the expected number of heads is $N / 2$. Bernoulli (1713) proved that $H / N$ converges to $1 / 2$ as $N$ goes to infinity. This was the first example of a law of large numbers. Of course, this does not mean that if you flip the coin 1000 times, you will see exactly 500 heads. Indeed, in $N$ coin flips one expects the number of heads to vary randomly around the value $N / 2$ in the scale $\sqrt{N}$. Moreover, for all $x \in$ $\mathbb{R}$,

$$
\lim _{N \rightarrow \infty} \mathbb{P}\left(H<\frac{1}{2} N+\frac{1}{2} \sqrt{N} x\right)=\int_{-\infty}^{x} \frac{e^{-y^{2} / 2}}{\sqrt{2 \pi}} d y .
$$

De Moivre (1738), Gauss (1809), Adrain (1809), and Laplace (1812) all participated in the proof of this result. The limiting distribution is known as the Gaussian (or sometimes normal or bell curve) distribution.

A proof of this follows from asymptotics of $n !$, as derived by de Moivre (1721) and named after Stirling (1729). Write

$$
n !=\Gamma(n+1)=\int_{0}^{\infty} e^{-t} t^{n} d t=n^{n+1} \int_{0}^{\infty} e^{n f(z)} d z,
$$

where $f(z)=\log z-z$ and the last equality is from the change of variables $t=n z$. The integral is dominated, as $n$ grows, by the maximal value of $f(z)$ on the interval $[0, \infty)$. This occurs at $z=1$; thus, expanding $f(z) \approx-1-\frac{(z-1)^{2}}{2}$ and plugging this into the integral yields the final expansion

$$
n ! \approx n^{n+1} e^{-n} \sqrt{2 \pi / n} .
$$


This general route of writing exact formulas for probabilities in terms of integrals and then performing asymptotics is quite common to the analysis of integrable models in the KPZ universality class - though those formulas and analyses are considerably more involved.

The universality of the Gaussian distribution was not broadly demonstrated until work of Chebyshev, Markov and Lyapunov around 1900. The central limit theorem (CLT) showed that the exact nature of coin flipping is immaterial any sum of independent, identically distributed (iid) random variables with finite mean and variance will demonstrate the same limiting behaviour.

Theorem 2.1. Let $X_{1}, X_{2}, \ldots$ be iid random variables of finite mean $m$ and variance $v$. Then, for all $x \in \mathbb{R}$,

$$
\lim _{N \rightarrow \infty} \mathbb{P}\left(\sum_{i=1}^{N} X_{i}<m N+v \sqrt{N} x\right)=\int_{-\infty}^{x} \frac{e^{-y^{2} / 2}}{\sqrt{2 \pi}} d y .
$$

Proofs of this result use different tools than the exact analysis of coin flipping and much of probability theory deals with the study of Gaussian processes that arise through various generalisations of the CLT. The Gaussian distribution is ubiquitous and, as it is the basis for much of classical statistics and thermodynamics, it has had immense societal impact.

\section{$3 \quad$ Random versus ballistic deposition}

The random deposition model is one of the simplest (and least realistic) models for a randomly growing one-dimensional interface. Unit blocks fall independently and in parallel from the sky above each site of $\mathbb{Z}$ according to exponentially distributed waiting times (see Figure 1). Recall that a random variable $X$ has an exponential distribution of rate $\lambda>0$ (or mean $1 / \lambda)$ if $\mathbb{P}(X>x)=e^{-\lambda x}$. Such random variables are characterised by the memoryless property - conditioned on the event that $X>x, X-x$ still has the exponential distribution of the same rate. Consequently, the random deposition model is Markov - its future evolution only depends on the present state (and not on its history).

The random deposition model is quite simple to analyse since each column grows independently. Let $h(t, x)$ record the height above site $x$ at time $t$ and assume $h(0, x) \equiv 0$. Define random waiting times $w_{x, i}$ to be the time for the $i$-th block in column $x$ to fall. For any $n$, the event $h(t, x)<n$ is equivalent to $\sum_{i=1}^{n} w_{x, i}>t$. Since the $w_{x, i}$ are iid, the law of large numbers and central limit theory apply here. Assuming $\lambda=1$,

$$
\lim _{t \rightarrow \infty} \frac{h(t, x)}{t}=1, \quad \text { and } \quad \lim _{t \rightarrow \infty} \frac{h(t, x)-t}{t^{1 / 2}} \Rightarrow N(x)
$$

jointly over $x \in \mathbb{Z}$, where $\{N(x)\}_{x \in \mathbb{Z}}$ is a collection of iid standard Gaussian random variables. The top of Figure 2 shows a simulation of the random deposition model. The linear growth speed and lack of spatial correlation are quite evident. The fluctuations of this model are said to be in the Gaussian universality class since they grow like $t^{1 / 2}$, with Gaussian limit law and trivial transversal correlation length scale $t^{0}$. In general, fluctuation and transversal correlation exponents, as well as limiting distributions, constitute the description of a universality class and all models that match these limiting behaviours are said to lie in the same universality class.

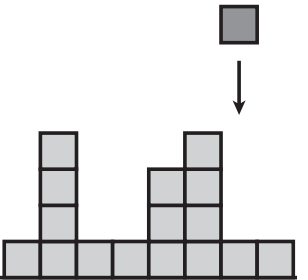

(a)

(b)

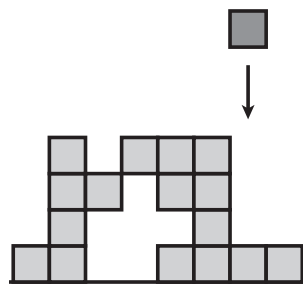

(c)

Figure 1. (A) and (B) illustrate the random deposition model and (C) and (D) illustrate the ballistic deposition model. In both cases, blocks fall from above each site with independent, exponentially distributed waiting times. In the first model, they land at the top of each column whereas in the second model they stick to the first edge to which they become incident.

While the Gaussian behaviour of this model is resilient against changes in the distribution of the $w_{x, i}$ (owing to the CLT), generic changes in the nature of the growth rules shatter the Gaussian behaviour. The ballistic deposition (or sticky block) model was introduced by Vold (1959) and, as one expects in real growing interfaces, displays spatial correlation. As before, blocks fall according to iid exponential waiting times; however, a block will now stick to the first edge against which it becomes incident. This mechanism is illustrated in Figure 1. This creates overhangs and we define the height function $h(t, x)$ as the maximal height above $x$ that is occupied by a box. How does this microscopic change manifest itself over time?

It turns out that sticky blocks radically change the limiting behaviour of this growth process. The bottom of Figure 2 records one simulation of the process. Seppäläinen (1999) gave a proof that there is still an overall linear growth rate. Moreover, by considering a lower bound by a width two system, one can see that this velocity exceeds that of the random deposition model. The exact value of this rate, however, remains unknown.

The simulation in Figure 2 (as well as the longer time results displayed in Figure 3) also shows that the scale of fluctuations of $h(t, x)$ is smaller than in random deposition and that the height function remains correlated transversally over a long distance. There are exact conjectures for these fluctuations. They are supposed to grow like $t^{1 / 3}$ and demonstrate a non-trivial correlation structure in a transversal scale of $t^{2 / 3}$. Additionally, precise predictions exist for the limiting distributions. Up to certain (presently undetermined) constants $c_{1}, c_{2}$, the sequence of scaled heights $c_{2} t^{-1 / 3}\left(h(t, 0)-c_{1} t\right)$ should converge to the so-called Gaussian Orthogonal Ensemble (GOE) Tracy-Widom distributed random variable. The Tracy-Widom distributions can be thought of as modern-day bell curves and their names GOE or GUE (for Gaussian 

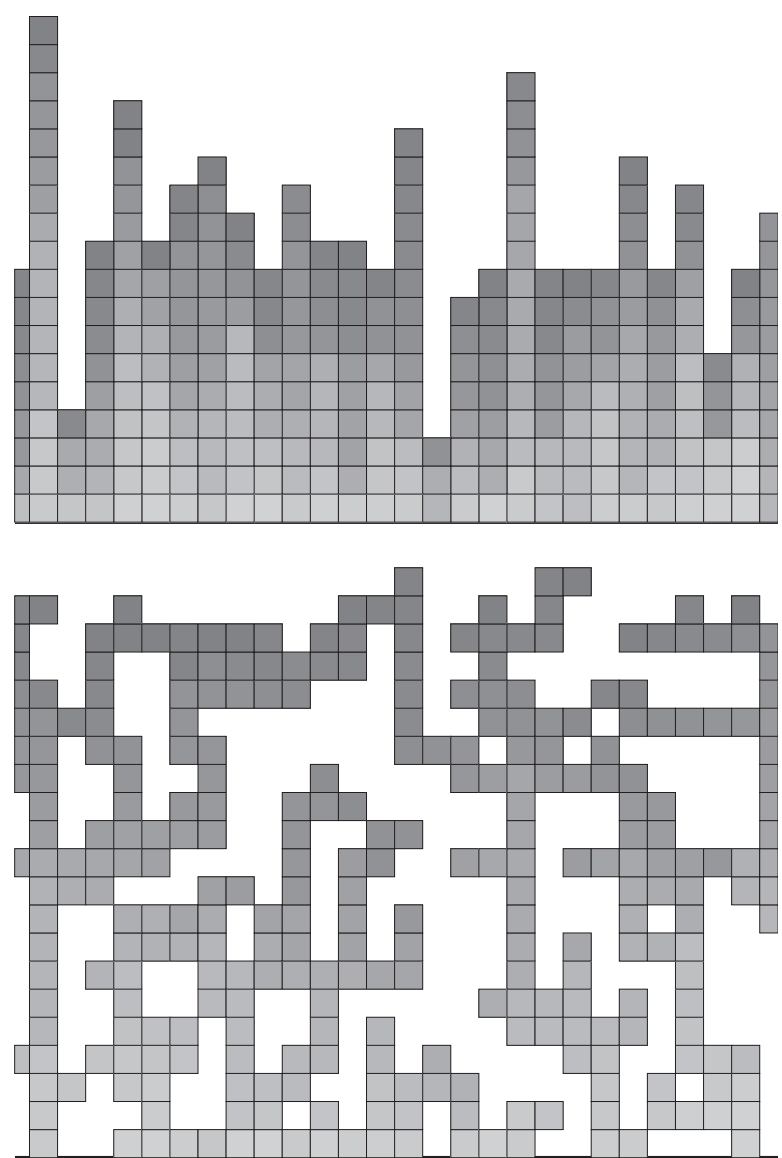

Figure 2. Simulation of random (top) versus ballistic (bottom) deposition models driven by the same process of falling blocks. The ballistic model grows much faster and has a smoother, more spatially correlated top interface.

Unitary Ensemble) come from the random matrix ensembles in which these distributions were first observed by TracyWidom $(1993,1994)$.

Ballistic deposition does not seem to be an integrable probabilistic system so where do these precise conjectures come from? The exact predictions come from the analysis of a few similar growth processes that just happen to be integrable! Ballistic deposition shares certain features with these models that are believed to be key for membership in the KPZ class:

- Locality: height function change depends only on neighbouring heights.

- Smoothing: large valleys are quickly filled in.

- Non-linear slope dependence: vertical effective growth rate depends non-linearly on local slope.

- $\quad$ Space-time independent noise: growth is driven by noise, which quickly decorrelates in space and time and does not display heavy tails.

It should be made clear that a proof of the KPZ class behaviour for the ballistic deposition model is far beyond what can be done mathematically (though simulations strongly suggest that the above conjecture is true).
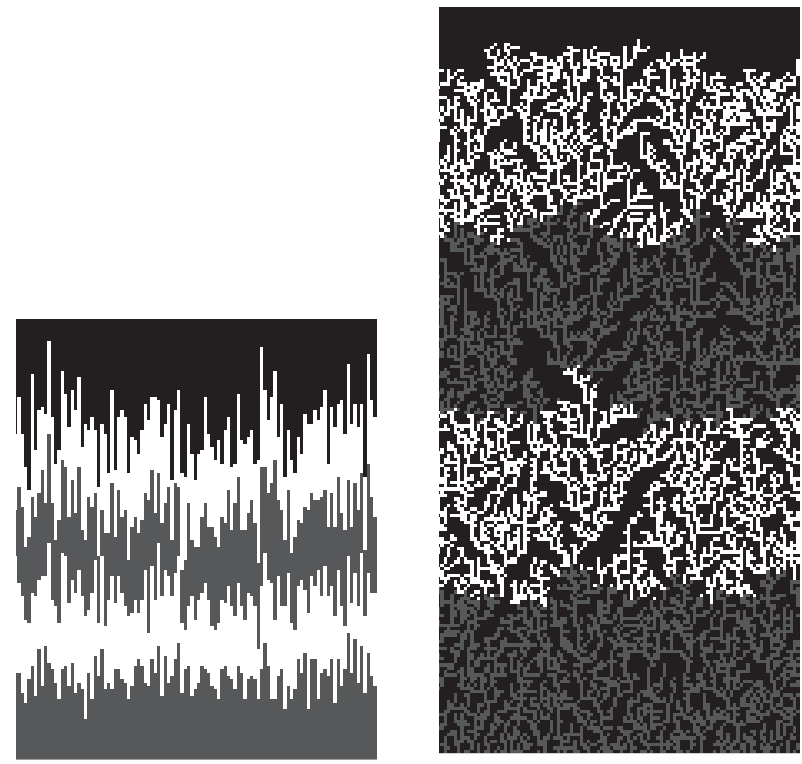

Figure 3. Simulation of random (left) versus ballistic (right) deposition models driven by the same process of falling blocks and run for a long time. The white and grey colours represent different epochs of time in the simulation. The sizes of the boxes in both figures are the same.

\section{Corner growth model}

We come to the first example of an integrable probabilistic system in the KPZ universality class - the corner growth model. The randomly growing interface is modelled by a height function $h(t, x)$ that is continuous, piecewise linear and composed of $\sqrt{2}$-length line increments of slope +1 or -1 , changing value at integer $x$. The height function evolves according to the Markovian dynamics that each local minimum of $h$ (looking like $\vee$ ) turns into a local maximum (looking like $\wedge$ ) according to an exponentially distributed waiting time. This happens independently for each minimum. This change in height function can also be thought of as adding boxes (rotated by $45^{\circ}$ ). See Figures 4 and 5 for further illustration of this model.

Wedge initial data means that $h(0, x)=|x|$ while flat initial data (as considered for ballistic deposition) means that $h(0, x)$ is given by a periodic saw-tooth function that goes between heights 0 and 1 . We will focus on wedge initial data. Rost (1980) proved a law of large numbers for the growing interface when time, space and the height function are scaled by the same large parameter $L$.
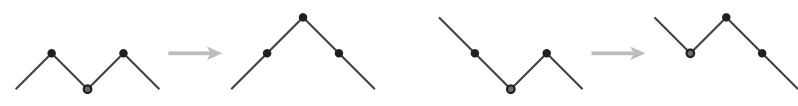

(a)

(b)
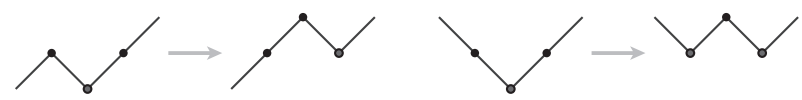

(c)

(d)

Figure 4. Various possible ways that a local minimum can grow into a local maximum. 

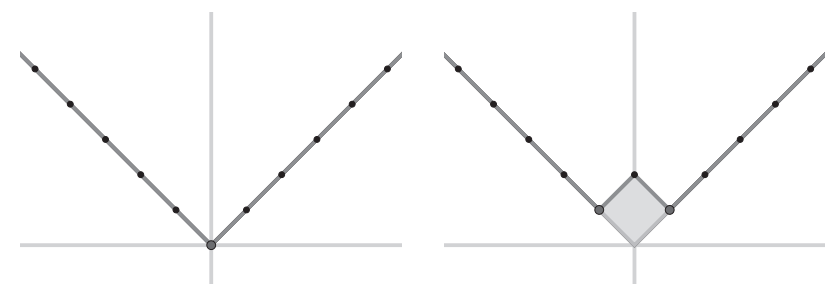

(a)

(b)
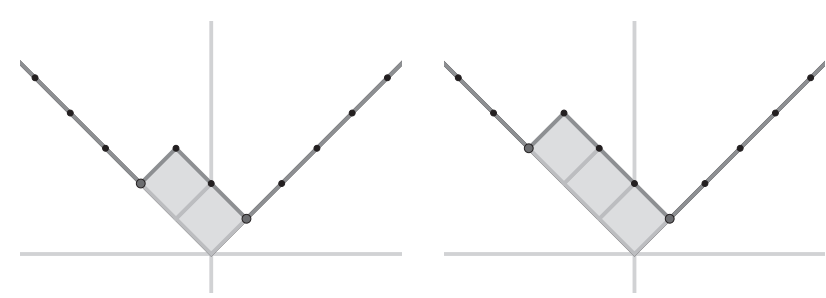

(c)

(d)

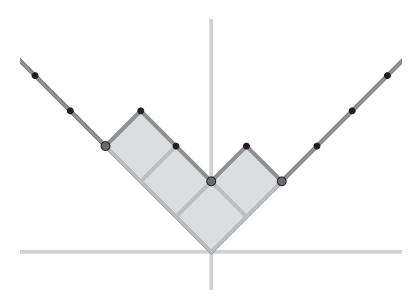

(e)

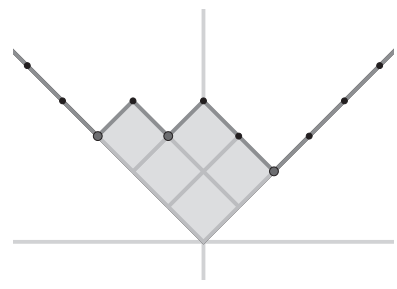

(f)
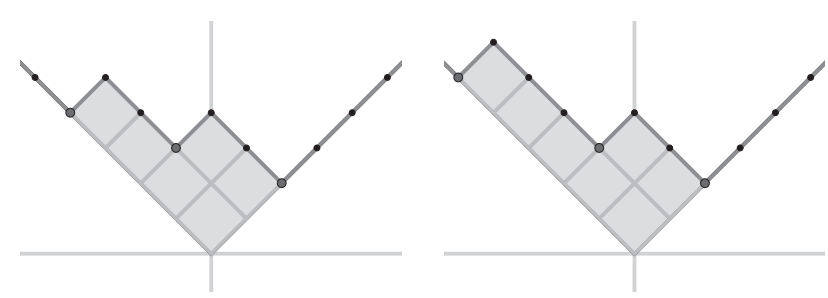

(g)

(h)

Figure 5. The corner growth model starts with an empty corner, as in (A). There is only one local minimum (the large dot) and after an exponentially distributed waiting time, this turns into a local maximum by filling in the site above it with a block, as in (B). In (B), there are now two possible locations for growth (the two dots). Each one has an exponentially distributed waiting time. (C) corresponds to the case when the left local minimum grows before the right one. By the memoryless property of exponential random variables, once in state $(C)$, we can think of choosing new exponentially distributed waiting times for the possible growth destinations. Continuing in a similar manner, we arrive at the evolution in (D) through (H).

Theorem 4.1. For wedge initial data,

$$
\lim _{L \rightarrow \infty} \frac{h(L t, L x)}{L}=\mathfrak{h}(t, x):= \begin{cases}t \frac{1-(x / t)^{2}}{2} & |x|<t, \\ |x| & |x| \geq t .\end{cases}
$$

Figure 6 displays the result of a computer simulation wherein the limiting parabolic shape is evident. The function $\mathfrak{h}$ is the unique viscosity solution to the Hamilton-Jacobi equation

$$
\frac{\partial}{\partial t} \mathfrak{h}(t, x)=\frac{1}{2}\left(1-\left(\frac{\partial}{\partial x} \mathfrak{h}(t, x)\right)^{2}\right) .
$$
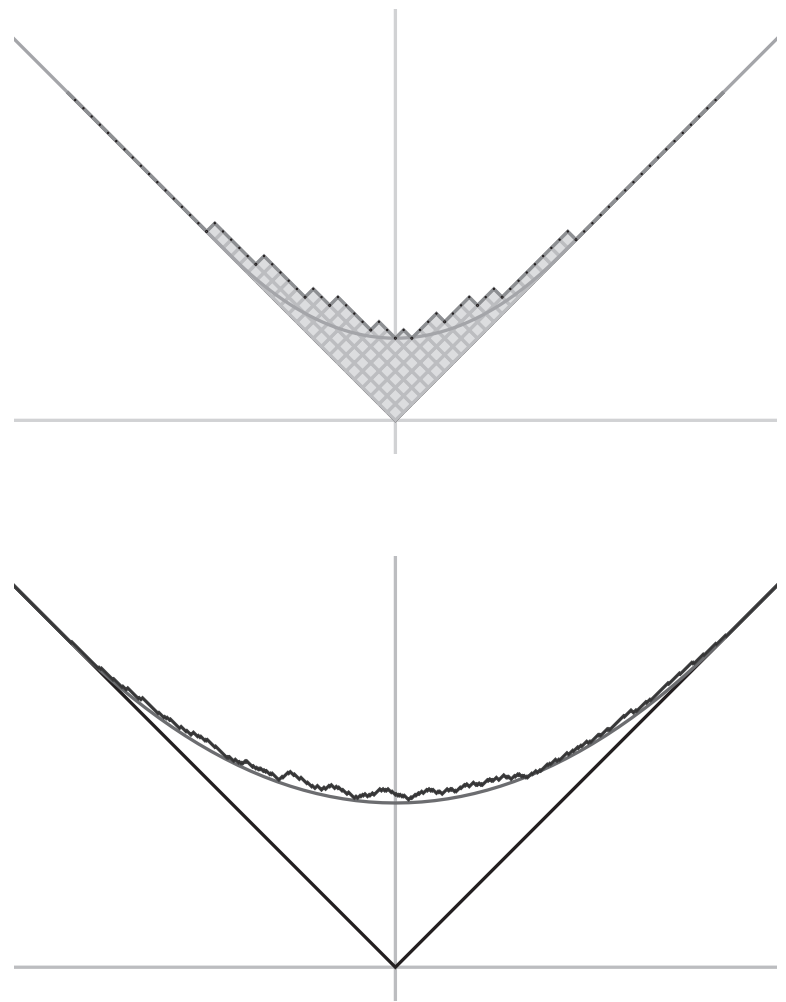

Figure 6. Simulation of the corner growth model. The top shows the model after a medium amount of time and the bottom shows it after a longer amount of time. The rough interface is the simulation while the smooth curve is the limiting parabolic shape. The simulation curve has vertical fluctuations of order $t^{1 / 3}$ and decorrelates spatially on distances of order $t^{2 / 3}$

This equation actually governs the evolution of the law of large numbers from arbitrary initial data.

The fluctuations of this model around the law of large numbers are what is believed to be universal. Figure 6 shows that the interface fluctuates around its limiting shape on a fairly small scale, with transversal correlation on a larger scale. For $\epsilon>0$, define the scaled and centred height function

$$
h_{\epsilon}(t, x):=\epsilon^{b} h\left(\epsilon^{-z} t, \epsilon^{-1} x\right)-\frac{\epsilon^{-1} t}{2},
$$

where the dynamic scaling exponent $z=3 / 2$ and the fluctuation exponent $b=1 / 2$. These exponents are easily remembered since they correspond with scaling time : space : fluctuations like $3: 2: 1$. These are the characteristic exponents for the KPZ universality class. Johansson (1999) proved that for fixed $t$, as $\epsilon \rightarrow 0$, the random variable $h_{\epsilon}(t, 0)$ converges to a GUE Tracy-Widom distributed random variable (see Figure 7). Results for the related model of the longest increasing subsequence in a random permutation were provided around the same time by Baik-Deift-Johansson (1999). For that related model, two years later, Prähofer-Spohn (2001) computed the analogue to the joint distribution of $h_{\epsilon}(t, x)$ for fixed $t$ and varying $x$.

The entire scaled growth process $h_{\epsilon}(\cdot, \cdot)$ should have a limit as $\epsilon \rightarrow 0$ that would necessarily be a fixed point under $3: 2: 1$ scaling. The existence of this limit (often called the $K P Z$ fixed point) remains conjectural. Still, much is known about the properties this limit should enjoy. It should be a stochastic process whose evolution depends on the limit of 


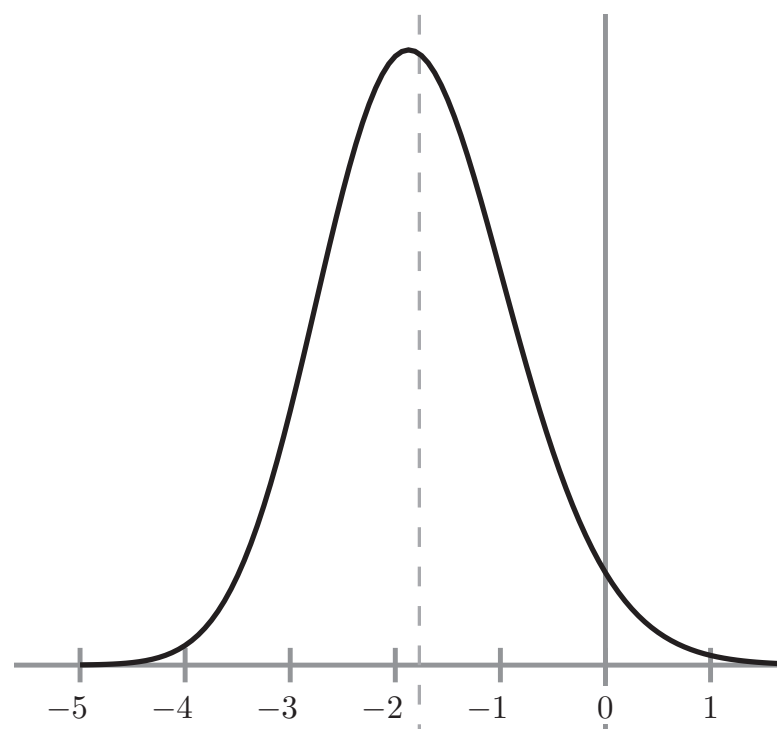

|

Figure 7. The density (top) and log of the density (bottom) of the GUE Tracy-Widom distribution. Though the density appears to look like a bell-curve (or Gaussian), this comparison is misleading. The mean and variance of the distribution are approximately -1.77 and 0.81 . The tails of the density (as shown in terms of the log of the density in the bottom plot) decay like $e^{-c_{-}|x|^{3}}$ for $x \ll 0$ and like $e^{-c_{+} x^{3 / 2}}$ for $x \gg 0$, for certain positive constants $c_{-}$and $c_{+}$. The Gaussian density decays like $e^{-c x^{2}}$ in both tails, with the constant $c$ related to the variance.

the initial data under the same scaling. The one-point distribution for general initial data, the multi-point and multi-time distribution for wedge initial data and various aspects of its continuity are all understood. Besides the existence of this limit, what is missing is a useful characterisation of the KPZ fixed point. Since the KPZ fixed point is believed to be the universal scaling limit of all models in the KPZ universality class and since corner growth enjoys the same key properties as ballistic deposition, one is also led to the conjecture that ballistic deposition scales to the same fixed point and hence enjoys the same scalings and limiting distributions. The reason why the GOE Tracy-Widom distribution came up in our earlier discussion is that we were dealing with flat rather than wedge initial data.

One test of the universality belief is to introduce partial asymmetry into the corner growth model. Now we change lo- cal minima into local maxima at rate $p$, and turn local maxima into local minima at rate $q$ (all waiting times are independent and exponentially distributed, and $p+q=1$ ). See 8 for an illustration of this partially asymmetric corner growth model. Tracy-Widom (2007-2009) showed that so long as $p>q$, the same law of large numbers and fluctuation limit theorem holds for the partially asymmetric model, provided that $t$ is replaced by $t /(p-q)$. Since $p-q$ represents the growth drift, one simply has to speed up to compensate for this drift being smaller.

Clearly, for $p \leq q$, something different must occur than for $p>q$. For $p=q$, the law of large numbers and fluctuations change nature. The scaling of time : space : fluctuations becomes $4: 2: 1$ and the limiting process under these scalings becomes the stochastic heat equation with additive white noise. This is the Edwards-Wilkinson (EW) universality class, which is described by the stochastic heat equation with additive noise. For $p<q$, the process approaches a stationary distribution where the probability of having $k$ boxes added to the empty wedge is proportional to $(p / q)^{k}$.

So, we have observed that for any positive asymmetry the growth model lies in the KPZ universality class while for zero asymmetry it lies in the EW universality class. It is natural to wonder whether by critically scaling parameters (i.e. $p-q \rightarrow$ 0 ), one might encounter a crossover regime between these two universality classes. Indeed, this is the case and the crossover is achieved by the KPZ equation that we now discuss.

\section{The KPZ equation}

The KPZ equation is written as

$$
\frac{\partial h}{\partial t}(t, x)=v \frac{\partial^{2} h}{\partial x^{2}}(t, x)+\frac{1}{2} \lambda\left(\frac{\partial h}{\partial x}(t, x)\right)^{2}+\sqrt{D} \xi(t, x),
$$

where $\xi(t, x)$ is Gaussian space-time white noise, $\lambda, v \in \mathbb{R}$, $D>0$ and $h(t, x)$ is a continuous function of time $t \in \mathbb{R}_{+}$ and space $x \in \mathbb{R}$, taking values in $\mathbb{R}$. Due to the white noise, one expects $x \mapsto h(t, x)$ to be only as regular as in Brownian motion. Hence, the non-linearity does not a priori make any sense (the derivative of Brownian motion has negative Hölder regularity). Bertini-Cancrini (1995) provided the physically relevant notion of solution (called the Hopf-Cole solution) and showed how it arises from regularising the noise, solving the (now well-posed) equation and then removing the noise and subtracting a divergence.

The equation contains the four key features mentioned earlier - the growth is local, depending on the Laplacian (smoothing), the square of the gradient (non-linear slope dependent growth) and white noise (space-time uncorrelated noise). Kardar, Parisi and Zhang introduced their eponymous equation and 3:2:1 scaling prediction in 1986 in an attempt to understand the scaling behaviours of random interface growth.

How might one see the 3:2:1 scaling from the KPZ equation? Define $h_{\epsilon}(t, x)=\epsilon^{b} h\left(\epsilon^{-z} t, \epsilon^{-1} x\right)$; then, $h_{\epsilon}$ satisfies the KPZ equation with scaled coefficients $\epsilon^{2-z} v, \epsilon^{2-z-b} \frac{1}{2} \lambda$ and $\epsilon^{b-\frac{z}{2}+\frac{1}{2}} \sqrt{D}$. It turns out that two-sided Brownian motion is stationary for the KPZ equation; hence, any non-trivial scaling must respect the Brownian scaling of the initial data and thus have $b=1 / 2$. Plugging this in, the only way to have 
no coefficient blow up to infinity and not to have every term shrink to zero (as $\epsilon \rightarrow 0$ ) is to choose $z=3 / 2$. This suggests the plausibility of the $3: 2: 1$ scaling. While this heuristic gives the right scaling, it does not provide for the scaling limit. The limit as $\epsilon \rightarrow 0$ of the equation (the inviscid Burgers equation where only the non-linearity survives) certainly does not govern the limit of the solutions. It remains something of a mystery as to exactly how to describe this limiting KPZ fixed point. The above heuristic says nothing of the limiting distribution of the solution to the KPZ equation and there does not currently exist a simple way to see what this should be.

It took just under 25 years until Amir-Corwin-Quastel (2010) rigorously proved that the KPZ equation is in the KPZ universality class. That work also computed an exact formula for the probability distribution of the solution to the KPZ equation - marking the first instance of a non-linear stochastic PDE for which this was accomplished. Tracy-Widom's work on the partially asymmetric corner growth model and the work of Bertini-Giacomin (1997) relating that model to the KPZ equation were the two main inputs in this development. See [3] for further details regarding this, as well as the simultaneous exact but non-rigorous steepest descent work of Sasamoto-Spohn (2010), and non-rigorous replica approach work of Calabrese-Le Doussal-Rosso (2010) and Dotsenko (2010).

The proof that the KPZ equation is in the KPZ universality class was part of an ongoing flurry of activity surrounding the KPZ universality class from a number of directions such as integrable probability [4], experimental physics [10] and stochastic PDEs. For instance, Bertini-Cancrini's Hopf-Cole solution relies upon a trick (the Hopf-Cole transform) that linearises the KPZ equation. Hairer (2011), who had been developing methods to make sense of classically ill-posed stochastic PDEs, focused on the KPZ equation and developed a direct notion of solution that agreed with the Hopf-Cole one but did not require use of the Hopf-Cole transform trick. Still, this does not say anything about the distribution of solutions or their long-time scaling behaviours. Hairer's KPZ work set the stage for his development of regularity structures in 2013 - an approach to construction solutions of certain types of illposed stochastic PDEs - work for which he was awarded a Fields Medal.

\section{$6 \quad$ Interacting particle systems}

There is a direct mapping (see Figure 8) between the partially asymmetric corner growth model and the partially asymmetric simple exclusion process (generally abbreviated ASEP). One can associate to every -1 slope line increment a particle on the site of $\mathbb{Z}$ above which the increment sits, and to every +1 slope line increment an empty site. The height function then maps onto a configuration of particles and holes on $\mathbb{Z}$, with at most one particle per site. When a minimum of the height function becomes a maximum, it corresponds to a particle jumping right by one into an empty site and, likewise, when a maximum becomes a minimum, a particle jumps left by one into an empty site. Wedge initial data for corner growth corresponds to having all sites to the left of the origin initially occupied and all to the right empty - this is often called step initial data due to the step function in terms of particle den-

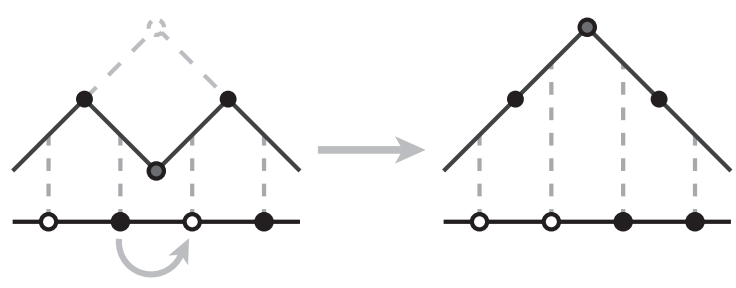

(a)

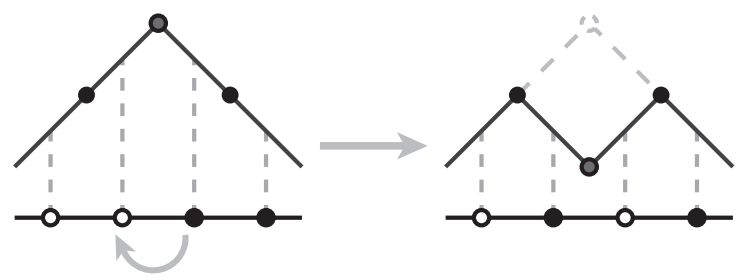

(b)

Figure 8. Mapping the partially asymmetric corner growth model to the partially asymmetric simple exclusion process. In (A), the local minimum grows into a local maximum. In terms of the particle process beneath it, the minimum corresponds to a particle followed by a hole and the growth corresponds to said particle jumping into the hole to its right. In (B), the opposite is shown as the local maximum shrinks into a local minimum. Correspondingly, there is a hole followed by a particle and the shrinking results in the particle moving into the hole to its left.

sity. ASEP was introduced in biology literature in 1968 by MacDonald-Gibbs-Pipkin as a model for RNA's movement during transcription. Soon after, it was independently introduced within the probability literature in 1970 by Spitzer.

The earlier quoted results regarding corner growth immediately imply that the number of particles to cross the origin after a long time $t$ demonstrates KPZ class fluctuation behaviour. KPZ universality would have that generic changes to this model should not change the KPZ class fluctuations. Unfortunately, such generic changes destroy the model's integrable structure. There are a few integrable generalisations discovered over the past five years that demonstrate some of the resilience of the KPZ universality class against perturbations.

TASEP (the totally asymmetric version of ASEP) is a very basic model for traffic on a one-lane road in which cars (particles) move forward after exponential rate one waiting times, provided the site is unoccupied. A more realistic model would account for the fact that cars slow down as they approach the one in front. The model of $q$-TASEP does just that (Figure 9). Particles jump right according to independent exponential waiting times of rate $1-q^{\text {gap }}$, where gap is the number of empty spaces to the next particle to the right. Here $q \in[0,1)$ is a different parameter than in the ASEP, though when $q$ goes to zero, these dynamics become those of TASEP.

Another feature one might include in a more realistic traf-

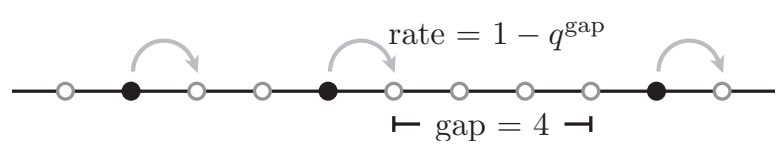

Figure 9. The $q$-TASEP, whereby each particle jumps one to the right after an exponentially distributed waiting time with rate given by $1-q^{\text {gap }}$. 


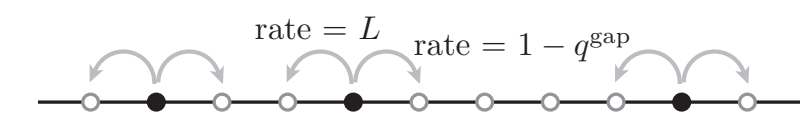

(a)

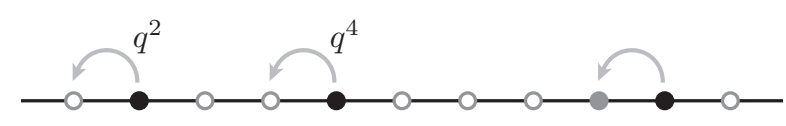

(b)

Figure 10. The $q$-pushASEP. As shown in (A), particles jump right according to the $q$-TASEP rates and left according to independent exponentially distributed waiting times of rate $L$. When a left jump occurs, it may trigger a cascade of left jumps. As shown in (B), the right-most particle has just jumped left by one. The next particle (to its left) instantaneously jumps left by one with probability given by $q^{\text {gap }}$, where gap is the number of empty sites between the two particles before the left jumps occurred (in this case gap $=4$ ). If that next left jump is realised, the cascade continues to the next-left particle according to the same rule, otherwise it stops and no other particles jump left in that instant of time.

fic model is the cascade effect of braking. The $q$-pushASEP includes this (Figure 10). Particles still jump right according to $q$-TASEP rules; however, particles may now also jump left after exponential rate $L$ waiting times. When such a jump occurs, it prompts the next particle to the left to likewise jump left, with a probability given by $q^{\text {gap }}$, where gap is the number of empty spaces between the original particle and its left neighbour. If that jump occurs, it may likewise prompt the next left particle to jump, and so on. Of course, braking is not the same as jumping backwards; however, if one goes into a moving frame, this left jump is like a deceleration. It turns out that both of these models are solvable via the methods of Macdonald processes as well as stochastic quantum integrable systems and it has thus been proved that, just as for ASEP, they demonstrate KPZ class fluctuation behaviour (see the review [4]).

\section{$7 \quad$ Paths in a random environment}

There is yet another class of probabilistic systems related to the corner growth model. Consider the totally asymmetric version of this model, starting from wedge initial data. An alternative way to track the evolving height function is to record the time when a given box is grown. Using the labelling shown in Figure 11, let us call $L(x, y)$ this time, for $x, y$ positive integers. A box $(x, y)$ may grow once its parent blocks $(x-1, y)$ and $(x, y-1)$ have both grown - though even then it must wait for an independent exponential waiting time that we denote by $w_{x, y}$. Thus, $L(x, y)$ satisfies the recursion

$$
L(x, y)=\max (L(x-1, y), L(x, y-1))+w_{x, y},
$$

subject to boundary conditions $L(x, 0) \equiv 0$ and $L(0, y) \equiv 0$. Iterating yields

$$
L(x, y)=\max _{\pi} \sum_{(i, j) \in \pi} w_{i, j}
$$

where the maximum is over all up-right and up-left lattice paths between box $(1,1)$ and $(x, y)$. This model is called last passage percolation with exponential weights. Following from the earlier corner growth model results, one readily sees that for any positive real $(x, y)$, for large $t, L(\lfloor x t\rfloor,\lfloor y t\rfloor)$

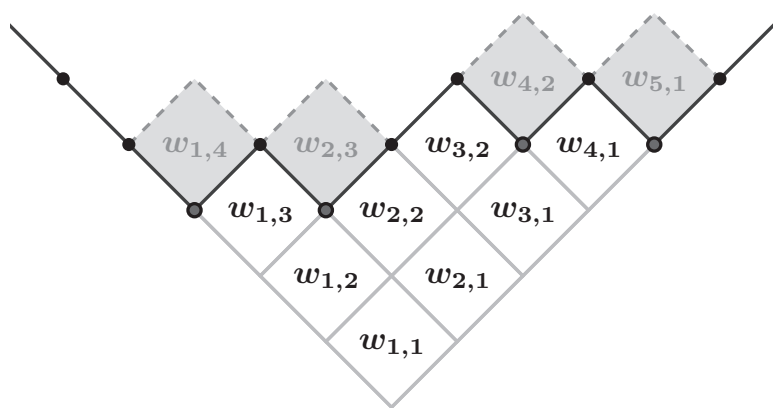

Figure 11. The relation between the corner growth model and last passage percolation with exponential weights. $w_{i, j}$ are the waiting times between when a box can grow and when it does grow. $L(x, y)$ is the time when box $(x, y)$ grows.

demonstrates KPZ class fluctuations. A very compelling and entirely open problem is to show that this type of behaviour persists when the distribution of $w_{i, j}$ is no longer exponential. The only other solvable case is that of geometric weights. A certain limit of the geometric weights leads to maximising the number of Poisson points along directed paths. Fixing the total number of points, this becomes equivalent to finding the longest increasing subsequence of a random permutation. The KPZ class behaviour for this version of last passage percolation was shown by Baik-Deift-Johansson (1999).

There is another related integrable model that can be thought of as describing the optimal way to cross a large grid with stop lights at intersections. Consider the first quadrant of $\mathbb{Z}^{2}$ and to every vertex $(x, y)$ assign waiting times to the edges leaving the vertex rightwards and upwards. With a probability of $1 / 2$, the rightward edge has waiting time zero, while the upward edge has waiting time given by exponential rate 1 random variables; otherwise, reverse the situation. The edge waiting time represents the time needed to cross an intersection in the given direction (the walking time between lights has been subtracted). The minimal passage time from $(1,1)$ to $(x, y)$ is given by

$$
P(x, y)=\min _{\pi} \sum_{e \in \pi} w_{e},
$$

where $\pi$ goes right or up in each step and ends on the vertical line above $(x, y)$ and $w_{e}$ is the waiting time for edge $e \in \pi$. From the origin, there will always be a path of zero waiting time, whose spatial distribution is that of the graph of a simple symmetric random walk. Just following this path, one can get very close to the diagonal $x=y$ without waiting. On the other hand, for $x \neq y$, getting to $(\lfloor x t\rfloor,\lfloor y t\rfloor)$ for large $t$ requires some amount of waiting. Barraquand-Corwin (2015) demonstrated that as long as $x \neq y, P(\lfloor x t\rfloor,\lfloor y t\rfloor)$ demonstrates KPZ class fluctuations. This should be true when $\pi$ is restricted to hit exactly $(x, y)$, though that result has not yet been proved. Achieving this optimal passage time requires some level of omnipotence as you must be able to look forward before choosing your route. As such, it could be considered as a benchmark against which to test various routing algorithms.

In addition to maximising or minimising path problems, the KPZ universality class describes fluctuations of "positive temperature' versions of these models in which energetic or probabilistic favouritism is assigned to paths based on the sum of space-time random weights along its graph. One such sys- 


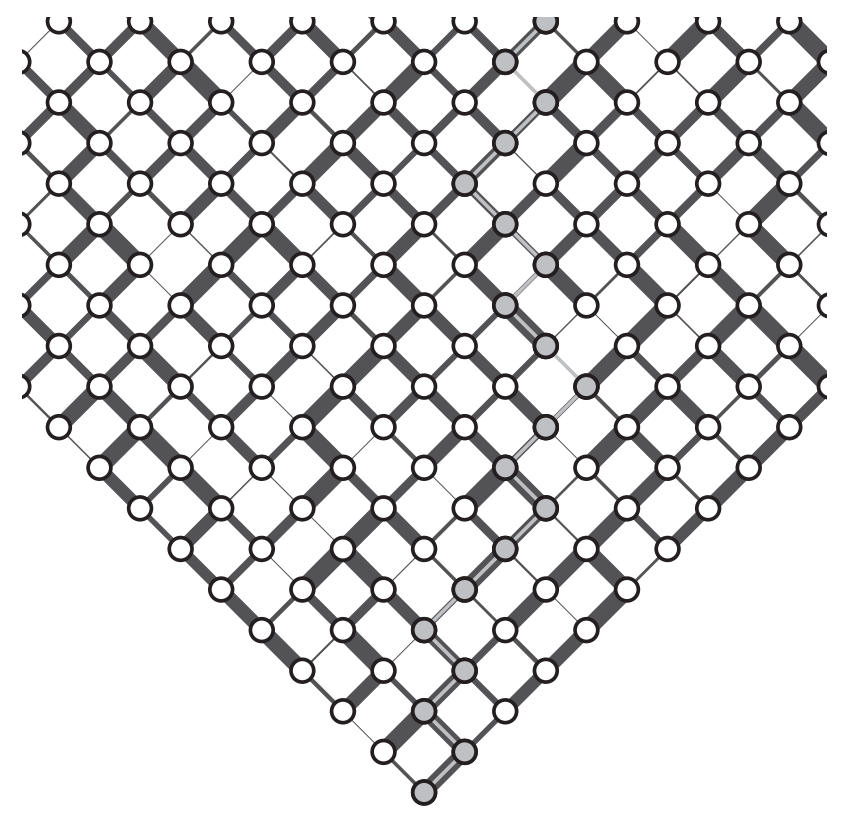

Figure 12. The random walk in a space-time random environment. For each pair of up-left and up-right pointing edges leaving a vertex $(y, s)$, the width of the edges is given by $u_{y, s}$ and $1-u_{y, s}$, where $u_{y, s}$ are independent uniform random variables on the interval $[0,1]$. A walker (the grey highlighted path) then performs a random walk in this environment, jumping up-left or up-right from a vertex with probability equal to the width of the edges.

tem is called directed polymers in random environment and is the detropicalisation of LPP where one replaces the operations of $(\max ,+)$ by $(+, \times)$ in the definition of $L(x, y)$. Then, the resulting (random) quantity is called the partition function for the model and its logarithm (the free energy) is conjectured for very general distributions on $w_{i, j}$ to show KPZ class fluctuations. There is one known integrable example of weights for which this has been proved - the inverse-gamma distribution, introduced by Seppäläinen (2009) and proved in the work of Corwin-O'Connell-Seppäläinen-Zygouras (2011) and Borodin-Corwin-Remenik (2012).

The stop light system discussed above also has a positive temperature lifting of which we will describe a special case (see Figure 12 for an illustration). For each space-time vertex $(y, s)$, choose a random variable $u_{y, s}$ distributed uniformly on the interval $[0,1]$. Consider a random walk $X(t)$ that starts at $(0,0)$. If the random walk is in position $y$ at time $s$ then it jumps to position $y-1$ at time $s+1$ with probability $u_{y, s}$ and to position $y+1$ with probability $1-u_{y, s}$. With respect to the same environment of $u$ 's, consider $N$ such random walks. The fact that the environment is fixed causes them to follow certain high probability channels. This type of system is called a random walk in a space-time random environment and the behaviour of a single random walker is quite well understood. Let us, instead, consider the maximum of $N$ walkers in the same environment $M(t, N)=\max _{i=1}^{N} X^{(i)}(t)$. For a given environment, it is expected that $M(t, N)$ will localise near a given random environment dependent value. However, as the random environment varies, this localisation value does as well in such a way that for $r \in(0,1)$ and large $t, M\left(t, e^{r t}\right)$ displays KPZ class fluctuations.

\section{$8 \quad$ Big problems}

It took almost 200 years from the discovery of the Gaussian distribution to the first proof of its universality (the central limit theorem). So far, KPZ universality has withstood proof for almost three decades and shows no signs of yielding.

Besides universality, there remain a number of other big problems for which little to no progress has been made. All of the systems and results discussed herein have been $1+1$ dimensional, meaning that there is one time dimension and one space dimension. In the context of random growth, it makes perfect sense (and is quite important) to study surface growth, i.e. $1+2$ dimensional. In the isotropic case (where the underlying growth mechanism is roughly symmetric with respect to the two spatial dimensions), there are effectively no mathematical results though numerical simulations suggest that the $1 / 3$ exponent in the $t^{1 / 3}$ scaling for corner growth should be replaced by an exponent of roughly 0.24 . In the anisotropic case there have been a few integrable examples discovered that suggest very different (logarithmic scale) fluctuations such as observed by Borodin-Ferrari (2008).

Finally, despite the tremendous success in employing methods of integrable probability to expand and refine the KPZ universality class, there still seems to be quite a lot of room to grow and new integrable structures to employ. Within the physics literature, there are a number of exciting new directions in which the KPZ class has been pushed, including out-of-equilibrium transform and energy transport with multiple conservation laws, front propagation equations, quantum localisation with directed paths and biostatistics. What is equally important is to understand what type of perturbations break out of the KPZ class.

Given all of the rich mathematical predictions, one might hope that experiments would have revealed KPZ class behaviour in nature. This is quite a challenge since determining scaling exponents and limiting fluctuations require immense numbers of repetitions of experiments. However, there have been a few startling experimental confirmations of these behaviours in the context of liquid crystal growth, bacterial colony growth, coffee stains and fire propagation (see [10] and references therein). Truly, the study of the KPZ universality class demonstrates the unity of mathematics and physics at its best.

\section{Acknowledgements}

The author appreciates comments on a draft of this article by A. Borodin, P. Ferrari and H. Spohn. This text is loosely based on his "Mathematic Park" lecture entitled Universal phenomena in random systems and delivered at the Institut Henri Poincaré in May 2015. The author is partially supported by the NSF grant DMS-1208998, by a Clay Research Fellowship, by the Poincaré Chair and by a Packard Fellowship for Science and Engineering.

\section{References}

[1] A. Barabasi, H. Stanley. Fractal concepts in surface growth. Cambridge University Press (1995).

[2] A. Borodin, V. Gorin. Lectures on integrable probability. arXiv:1212.3351. 
[3] I. Corwin. The Kardar-Parisi-Zhang equation and universality class. Rand. Mat.: Theo. Appl. 1:1130001 (2012).

[4] I. Corwin. Macdonald processes, quantum integrable systems and the Kardar-Parisi-Zhang universality class. Proceedings of the ICM (2014).

[5] T. Halpin-Healy, Y-C. Zhang. Kinetic roughening, stochastic growth, directed polymers and all that. Phys. Rep., 254:215415 (1995).

[6] J. Krug. Origins of scale invariance in growth processes. Advances in Physics, 46:139-282 (1997).

[7] P. Meakin. Fractals, scaling and growth far from equilibrium. Cambridge University Press (1998).

[8] J. Quastel, H. Spohn. The one-dimensional KPZ equation and its universality class. J. Stat. Phys., to appear.

[9] H. Spohn. Large scale dynamics of interacting particles. Springer, Heidelberg (1991).

[10] K. Takeuchi, T. Halpin-Healy. A KPZ cocktail - shaken, not stirred ... J. Stat. Phys. Online First (2015).

Ivan Corwin [ic2354@columbia.edu] is a professor of mathematics at Columbia University. He works in between prob- ability, mathematical physics and integrable systems, and is particularly known for his work on the Kardar-Parisi-Zhang equation and universality class. He obtained his PhD at the Courant Institute and was the first Schramm Memorial Postdoctoral Fellow at Microsoft Research and MIT. He has also held a Clay Research Fel-

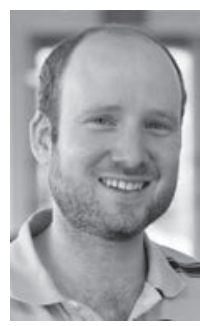
lowship and the first Poincare Chair at the Institute Henri Poincare, and currently holds a Packard Foundation Fellowship in Science and Engineering. He received the Young Scientist Prize, the Rollo Davidson Prize and was an invited speaker at the 2014 ICM.

This article has been reprinted with permission from the original publication in Notices of the American Mathematical Society 63 (2016), 230-239. Figures by Ivan Corwin and William Casselman, reprinted by permission of the creators. Picture of Ivan Corwin: Courtesy of Craig Tracy. 